\title{
STABLE BIRATIONAL EQUIVALENCE AND GEOMETRIC CHEVALLEY-WARNING
}

\author{
XIA LIAO
}

(Communicated by Lev Borisov)

\begin{abstract}
We propose a "geometric Chevalley-Warning" conjecture, that is, a motivic extension of the Chevalley-Warning theorem in number theory. Its statement is equivalent to a recent question raised by F. Brown and O. Schnetz. In this paper we show that the conjecture is true for linear hyperplane arrangements, quadratic and singular cubic hypersurfaces of any dimension, and cubic surfaces in $\mathbb{P}^{3}$. The last section is devoted to verifying the conjecture for certain special kinds of hypersurfaces of any dimension. As a by-product, we obtain information on the Grothendieck classes of the affine "Potts model" hypersurfaces considered by Aluffi and Marcolli.
\end{abstract}

\section{INTRODUCTION}

Let $\mathbb{F}_{q}$ be the finite field of $q$ elements with $q$ a prime power. The ChevalleyWarning theorem states that the number of solutions in $\mathbb{F}_{q}$ of a system of polynomial equations with $n$ variables is divisible by $q$, provided that the sum of the degrees of these polynomials is less than $n$. In [BS], $§ 3.3$, F. Brown and O. Schnetz ask a question in the Grothendieck ring of varieties $K_{0}($ Var $)$ : for what type of field $k$ is the class of an affine $k$-variety defined by equations satisfying the same degree condition as the Chevalley-Warning theorem a multiple of the class $\mathbb{L}$ of the affine line $\mathbb{A}_{k}^{1}$ ? In particular, is the statement true for algebraically closed fields? We propose the following variant of their question:

Conjecture 1 (Geometric Chevalley-Warning). Let $f_{1}, \ldots, f_{l}$ be homogeneous polynomials in $k\left[x_{0}, \ldots, x_{n}\right]$ such that $\sum_{i=1}^{l} \operatorname{deg}\left(f_{i}\right)<n+1$, where $k$ is an algebraically closed field of characteristic 0. Then $\left[Z\left(f_{1}, \ldots, f_{l}\right)\right] \equiv 1(\bmod \mathbb{L})$ in $K_{0}\left(\operatorname{Var}_{k}\right)$, where $Z\left(f_{1}, \ldots, f_{l}\right)$ denotes the set of zeros of $f_{1}, \ldots, f_{l}$ in $\mathbb{P}^{n}$.

Over a field $k$ as in the statement, the content of Conjecture 1 and the question of Brown-Schnetz are equivalent. Indeed, let $X=Z\left(f_{1}, \ldots, f_{l}\right) \subseteq \mathbb{P}^{n}$; then $[X]$. $(\mathbb{L}-1)+1$ is the class of the zero-locus of $f_{1}, \ldots, f_{l}$ in $\mathbb{A}^{n+1}$. An affirmative answer of the Brown-Schnetz question would imply that this class is $\equiv 0 \bmod \mathbb{L}$, and this is equivalent to $[X] \equiv 1 \bmod \mathbb{L}$.

In this paper, we show that Conjecture 1 is true for hyperplane arrangements, for quadratic hypersurfaces of any dimension, for cubic surfaces in $\mathbb{P}^{3}$, and for singular cubic hypersurfaces in any dimension.

Along the way, we also establish a result which settles some special cases of the conjecture in higher dimensions. The hypothesis that $k$ is an algebraically closed

Received by the editors January 30, 2012.

2010 Mathematics Subject Classification. Primary 14E08, 14N25, 14Q10. 
field of characteristic zero is used in our proofs, and it underlies the contextual remarks that follow in this introduction.

We note that the statement of Conjecture1 (for any $l$ ) is equivalent to the case of hypersurfaces $(l=1)$. Indeed, we have the equality $\left[Z\left(f_{1}, f_{2}\right)\right]=\left[Z\left(f_{1}\right)\right]+\left[Z\left(f_{2}\right)\right]-$ $\left[Z\left(f_{1} f_{2}\right)\right]$ in $K_{0}(\operatorname{Var})$, and the condition on degrees is satisfied by polynomials on one side of the equation whenever it is satisfied by polynomials on the other side of the equation. It follows that the conjecture is true for $Z\left(f_{1}, f_{2}\right)$ as long as it is true for the hypersurfaces $Z\left(f_{1}\right), Z\left(f_{2}\right)$, and $Z\left(f_{1} f_{2}\right)$. The same type of consideration applies to the zero set of any finite numbers of polynomial equations.

When the variety in consideration is a hypersurface, the condition on the degree asked by the geometric Chevalley-Warning conjecture becomes $\operatorname{deg}(f)<n+1$. This condition is reminiscent of results concerning the "weakened rationality" of varieties. Recall that a variety is rationally chain connected if two general points on the variety can be joined by a chain of rational curves. It is known that a smooth hypersurface of degree $d$ in $\mathbb{P}^{n}$ is rationally chain connected if and only if $d<n+1$ [KMM92. Moreover, if we fix the degree of the hypersurface and make the dimension of the ambient projective space large enough, then it is proved that such a general hypersurface is unirational HV92.

Introducing the notion of $\mathbb{L}$-rationality, Conjecture 1 admits an equivalent reformulation.

Definition 1.1 ([AM11]). A variety is $\mathbb{L}$-rational if its class in $K_{0}(\operatorname{Var})$ is 1 modulo $\mathbb{L}$.

Conjecture 2. Every hypersurface of degree $<n+1$ in $\mathbb{P}^{n}$ is $\mathbb{L}$-rational.

Conjecture 2 postulates that $\mathbb{L}$-rationality behaves in a sense as rational chain connectedness does. For instance, we know that neither cubic nor quartic smooth threefolds in $\mathbb{P}^{4}$ are rational, IM71, CG72], while they would both be $\mathbb{L}$-rational and rationally chain connected according to Conjecture 2 and the previous discussion.

The notion of $\mathbb{L}$-rationality is motivated by stable rationality. We recall that two nonsingular irreducible varieties $X$ and $Y$ are "stably birational" if $X \times \mathbb{P}^{k}$ is birational to $Y \times \mathbb{P}^{l}$ for some $k$ and $l$. We say that a nonsingular, complete irreducible variety is "stably rational" if it is stably birational to projective space. For nonsingular varieties, $\mathbb{L}$-rationality and stable rationality are equivalent. The argument is the following. Recall that the ideal generated by $\mathbb{L}$ in $K_{0}(V a r)$ has a concrete meaning in stably birational geometry. Denote by $\mathbb{Z}[S B]$ the monoid ring generated by stably birational classes of smooth complete irreducible varieties. Then M. Larsen and V. Lunts prove in LL03 that there exists a surjective homomorphism $\Psi_{S B}: K_{0}(V a r) \rightarrow \mathbb{Z}[S B]$, mapping the class of a smooth complete variety in $K_{0}(V a r)$ to its class in $\mathbb{Z}[S B]$, and that the kernel of this homomorphism is precisely $(\mathbb{L})$. Thus, a smooth projective variety is stably birational to projective space precisely when its class in $K_{0}(V a r)$ is 1 modulo $\mathbb{L}$.

The reader should note that, e.g., every cone is $\mathbb{L}$-rational; cf. Corollary 2.4. Also, according to the result we recalled above, a smooth projective rational variety is $\mathbb{L}$-rational. However, singular rational varieties may well not be $\mathbb{L}$-rational. For example, if the normalization morphism of an irreducible rational curve is not set theoretically injective, then the curve itself is not $\mathbb{L}$-rational. Thus, "most" singular rational curves are not $\mathbb{L}$-rational. Examples in higher dimension may be obtained 
by applying Lemma 3.3 , While all varieties considered in this paper are ruled or rational, it is by no means obvious a priori that they should be $\mathbb{L}$-rational as prescribed by Conjecture 1 and as we prove below.

We wrap up this discussion by noting that rationally chain connectedness admits a description analogous to the description of stable rationality we just recalled. In KR, B. Kahn and R. Sujatha construct a category of pure birational motives by localizing the category of pure motives with respect to certain classes of birational morphisms. They prove $(\underline{\mathrm{KR}}, \S 3.1)$ that if $X$ is a rationally chain connected smooth projective $F$-variety, then $h^{\circ}(X)=1$ in $\operatorname{Mot}_{\text {rat }}^{\circ}(F, \mathbb{Q})$. Thus, $h^{\circ}(X)$ plays for rational connectedness a role analogous to the role played by the class of $X$ in $\mathbb{Z}[S B] \cong K_{0}(\operatorname{Var}) /(\mathbb{L})$ for stable rationality.

This paper is a minor revision of a paper written by the author in the spring of 2011 and posted on the arXiv in October. We note that some of the results have also been obtained by Emel Bilgin in a paper posted in December ([Bil $)$.

\section{A feW SIMPle CASES OF The CONJECTURE}

In this section we verify that the conjecture is true when the degrees of the homogeneous polynomials defining the variety are low. Namely, we will show the following results:

Proposition 2.1. If $X$ is the union of $n$ or fewer hyperplanes in $\mathbb{P}^{n}$, then $X$ is $\mathbb{L}$-rational.

Proposition 2.2. Any quadratic hypersurface in $\mathbb{P}^{n}(n>1)$ is $\mathbb{L}$-rational.

Proposition 2.1 can be proved in a way similar to the reduction of the varieties in Conjecture 1 to hypersurfaces. In fact, the equation of $X$ can be written as $f_{1} \ldots f_{l}$, where $l$ is the number of hyperplanes and the $f_{i}$ 's are all linear equations. Then $[X]=Z\left[f_{1} \ldots f_{l-1}\right]+Z\left[f_{l}\right]-Z\left[f_{1} \ldots f_{l-1}, f_{l}\right]$. The notation $Z[\ldots]$ indicates the set of common zeros of the equations appearing in the bracket, separated by commas, as mentioned in Conjecture 1. The last term is the class of the union of $l-1$ hyperplanes in $\mathbb{P}^{n-1}$. By induction, all terms on the right side of the equation are equivalent to 1 modulo $\mathbb{L}$, so is $[X]$.

To prove Proposition 2.2, we observe that any singular quadratic hypersurface is a cone. According to the following lemma and its corollary, the singular case can be taken care of generally, and we are left to consider the class of a nonsingular quadratic hypersurface.

Lemma 2.3. Let $Z$ be the join of varieties $X$ and $Y$, which is obtained by connecting pairs of points from $X$ and $Y$ by $\mathbb{P}^{1}$ and assuming these rational curves only meet at points of $X$ or $Y$. If either $X$ or $Y$ is $\mathbb{L}$-rational, then $Z$ is also $\mathbb{L}$-rational.

Proof. Taking out $X$ and $Y$ from the variety $Z$, we get a bundle over $X \times Y$ whose fiber is the complement of 2 points in $\mathbb{P}^{1}$. Thus the class of $Z$ in $K_{0}($ Var $)$ is $[X] \cdot[Y] \cdot(\mathbb{L}-1)+[X]+[Y]=[X] \cdot[Y] \cdot \mathbb{L}-([X]-1) \cdot([Y]-1)+1$.

Corollary 2.4. If the projective variety $X^{\prime} \subset \mathbb{P}^{m}$ is a cone over another projective variety $X \subset \mathbb{P}^{n}, n<m$, then $X^{\prime}$ is $\mathbb{L}$-rational.

Remark 2.5. The union of $n$ or fewer hyperplanes in $\mathbb{P}^{n}$ is a cone (with its vertex at an arbitrary point in the set of the intersection). Thus we get a new proof of Proposition 2.1 
A special case of the result by Larsen-Lunts mentioned in the introduction gives an effective treatment of the Chevalley-Warning problem for nonsingular quadratic hypersurfaces, as

Lemma 2.6. Every rational smooth complete variety is $\mathbb{L}$-rational.

Proof. A rational smooth complete variety has the same stable birational class as a point. Thus the difference of its class in $K_{0}(V a r)$ and 1 is in the ideal generated by $\mathbb{L}$ LL03].

The previous lemmas settle the Chevalley-Warning problem for quadratic hypersurfaces. However, we can give another proof for the nonsingular one avoiding the use of Lemma 2.6. Let $Q$ be a nonsingular quadratic hypersurface. The projection from the "north pole" gives a nice birational map between $Q$ and $\mathbb{A}^{n}$ which allows us to stratify $Q$.

Proof of Proposition 2.2 in the nonsingular case. First, let's fix some notation. Let $Q_{n}$ be the nonsingular quadratic hypersurface in $\mathbb{P}^{n+1}$ defined by the equation $X_{0}^{2}+X_{1}^{2}+\ldots+X_{n+1}^{2}=0$ and $Y_{n}$ be the affine variety defined by $\sum_{i=1}^{n+1} y_{i}^{2}=1$ in $\mathbb{A}^{n+1}$.

Projecting from the point $P=(0,0, \ldots, 1)$, we can establish a birational map between $Y_{n}$ and $\mathbb{A}^{n}$. The formula is given by

$$
x_{i}=-\frac{y_{i}}{y_{n+1}-1} .
$$

Here, the $x_{i}$ 's are coordinates of the affine space $\mathbb{A}^{n}$.

The inverse rational map from $\mathbb{A}^{n}$ to $Q_{n}$ is given by the formula

$$
y_{i}=\frac{2 x_{i}}{\sum_{i=1}^{n} x_{i}^{2}+1} \quad y_{n+1}=\frac{\sum_{i=1}^{n} x_{i}^{2}-1}{\sum_{i=1}^{n} x_{i}^{2}+1} .
$$

From this description, it is easy to see that the closed set $Z\left(\sum x_{i}^{2}+1\right)$ is not in the image of the polar projection. Then we have the following relation in $K_{0}($ Var $)$ :

$$
\left[Y_{n}\right]-\left[Z\left(\sum_{i=1}^{n} y_{i}^{2}\right)\right]=\left[\mathbb{A}^{n}\right]-\left[Y_{n-1}\right] .
$$

In addition to this relation, we also have the trivial relation

$$
\left[Q_{n}\right]=\left[Q_{n-1}\right]+\left[Y_{n}\right]
$$

Because the variety $Z\left(\sum_{i=1}^{n} y_{i}^{2}\right)$ is a cone, by the proof of Corollary 2.4, we get that $Z\left(\sum_{i=1}^{n} y_{i}^{2}\right)$ is equal to $1+(\mathbb{L}-1)\left[Q_{n-2}\right]$ in $K_{0}(V a r)$. So we can replace our first equation by

$$
\left[Y_{n}\right]-\left(1+(\mathbb{L}-1)\left[Q_{n-2}\right]\right)=\left[\mathbb{A}^{n}\right]-\left[Y_{n-1}\right] .
$$

With the last two equations and the simple cases $\left[Q_{1}\right]=\mathbb{L}+1,\left[Y_{0}\right]=2,\left[Y_{1}\right]=\mathbb{L}-1$, we can conclude by an induction on dimension that $\left[Q_{n}\right] \equiv 1(\bmod \mathbb{L})$ when $n>0$ and $\left[Y_{n}\right] \equiv 0(\bmod \mathbb{L})$ when $n>1$. 


\section{Cubic hypersurfaces}

The next easiest case to consider is the variety defined by a cubic equation in $\mathbb{P}^{3}$. We have the following theorem:

Theorem 3.1. Any cubic surface in $\mathbb{P}^{3}$ is $\mathbb{L}$-rational.

In fact, we can prove something more:

Theorem 3.2. Any singular cubic hypersurface in $\mathbb{P}^{n}(n \geqslant 3)$ is $\mathbb{L}$-rational.

The following lemma helps to analyze singular cubic hypersurfaces.

Lemma 3.3. Let $X \subseteq \mathbb{P}^{n}$ have equation $F=x_{n} f_{k}\left(x_{0}, \ldots, x_{n-1}\right)+f_{k+1}\left(x_{0}, \ldots\right.$, $\left.x_{n-1}\right)=0$, where $f_{k}$ and $f_{k+1}$ are homogeneous polynomials of degree $k$ and $k+1$

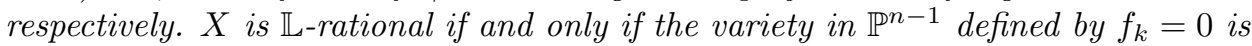
$\mathbb{L}$-rational.

Proof of Lemma 3.3. On the hypersurface $Z(F)$, the equation $f_{k}=0$ defines a cone with its vertex at the point $(0, \ldots, 1)$. By Corollary 2.4 this subvariety of the hypersurface $Z(F)$ is $\mathbb{L}$-rational. On the other hand, we have the isomorphism between the affine open set $f_{k} \neq 0$ in $\mathbb{P}^{n-1}$ and $Z(F)-Z\left(f_{k}\right)$ provided by $\left(x_{0}, \ldots, x_{n-1}\right) \rightarrow\left(x_{0}, \ldots, x_{n-1},-\frac{f_{k+1}}{f_{k}}\right)$. We see that the $\mathbb{L}$-rationality of $X$ is equivalent to the condition that the class of the affine open set $f_{k} \neq 0$ is in the ideal generated by $\mathbb{L}$. This happens if and only if the hypersurface $f_{k}=0$ in $\mathbb{P}^{n-1}$ is $\mathbb{L}$-rational.

Proof of Theorem 3.2. For a singular cubic hypersurface, we can assume one of its singular points is $(0: \ldots: 0: 1)$ by a change of projective coordinates. We keep using the notation of the previous lemma. The equation of the cubic surface is written as $x_{n} f_{2}\left(x_{0}, \ldots, x_{n-1}\right)+f_{3}\left(x_{0}, \ldots, x_{n-1}\right)=0$ or $f_{3}\left(x_{0}, \ldots, x_{n-1}\right)=0$, depending on whether the singular point is a double point or a triple point. Then the $\mathbb{L}$ rationality of the singular cubic hypersurface follows immediately from Lemma 3.3 , Proposition 2.2 or Corollary 2.4.

Proof of Theorem 3.1. A nonsingular cubic surface arises from the blow-up of $\mathbb{P}^{2}$ at 6 general points. In particular, it is rational. The $\mathbb{L}$-rationality follows from Lemma 2.6. The singular case has been covered by Theorem 3.2 .

Remark 3.4. One can also approach Theorem 3.1 by the classification of cubic surfaces given in [BW79]. That is, one can directly compute their class in $K_{0}(V a r)$ according to the standard equations given in this reference. This however leads to a lengthy computation.

Remark 3.5. The criterion we derived in Lemma 3.3 can be applied to give another proof of Proposition 2.2. As long as the rank of the quadratic form is greater than or equal to 2 , the equation can be written as $F=x_{0} x_{1}+x_{2}^{2}+\ldots$.

Corollary 3.6. If a singular quartic hypersurface in $\mathbb{P}^{4}$ has a triple point, then it is $\mathbb{L}$-rational.

Proof. Assuming the triple point is $(0: 0: 0: 0: 1)$, the equation of the quartic hypersuface can be written as $F=x_{4} f_{3}\left(x_{0}, x_{1}, x_{2}, x_{3}\right)+g_{4}\left(x_{0}, x_{1}, x_{2}, x_{3}\right)$. The $\mathbb{L}$-rationality follows immediately from Lemma 3.3 and Theorem 3.1 


\section{L-RATIONALITY OF HIGHER DIMENSIONAL VARIETIES}

Theorem 4.1. If the equation of a hypersurface of degree $n$ in $\mathbb{P}^{m}(m \geqslant n \geqslant 4)$ can be written as $F=x_{n} \ldots x_{4} f_{3}\left(x_{0}, x_{1}, x_{2}, x_{3}\right)+\sum_{i=5}^{n} x_{n} \ldots x_{i} g_{i-1}\left(x_{0}, \ldots, x_{i-2}\right)+$ $g_{n}\left(x_{0}, \ldots, x_{n-1}\right)$, then this hypersurface is $\mathbb{L}$-rational.

Proof. When $m>n$, not all coordinates of $\mathbb{P}^{m}$ appear in $F$. In this case $F$ defines a cone in $\mathbb{P}^{m}$ and the $\mathbb{L}$-rationality of this hypersurface follows from Corollary 2.4. When $m=n$, rewrite the polynomial as

$$
\begin{aligned}
F=x_{n}\left[x_{n-1} \ldots x_{4} f_{3}\left(x_{0}, x_{1}, x_{2}, x_{3}\right)\right. & +\sum_{i=5}^{n-1} x_{n-1} \ldots x_{i} g_{i-1}\left(x_{0}, \ldots, x_{i-2}\right) \\
& \left.+g_{n-1}\left(x_{0}, \ldots, x_{n-2}\right)\right]+g_{n}\left(x_{0}, \ldots, x_{n-1}\right) .
\end{aligned}
$$

Then the proof follows by induction, Lemma 3.3 and Corollary 3.6

Theorem 4.2. If the equation of the hypersurface of degree at most $n$ in $\mathbb{P}^{n}(n \geqslant 4)$ has degree 1 in all variables except at most 4 variables, then this hypersurface is $\mathbb{L}$-rational.

Proof. Suppose the 4 possibly nonlinear variables are $x_{0}, x_{1}, x_{2}$, and $x_{3}$. The proof proceeds by an induction on $n$. When $n=4$, the equation of the hypersurface is either $x_{4} f_{k}\left(x_{0}, x_{1}, x_{2}, x_{3}\right)+f_{k+1}\left(x_{0}, x_{1}, x_{2}, x_{3}\right)=0(k \leqslant 3)$ or $f_{k}\left(x_{0}, x_{1}, x_{2}, x_{3}\right)=$ $0(k \leqslant 4)$. Since we have checked the $\mathbb{L}$-rationality of cubic surfaces and quadratic hypersurfaces, the $\mathbb{L}$-rationality of such hypersurfaces are guaranteed by Lemma 3.3 or Corollary 2.4. When $n>4$, if the equation of the hypersurface is written only in terms of $x_{0}, x_{1}, x_{2}, x_{3}$, then it is a cone. Otherwise, let $x_{n}$ be one of its linear variables. Then the equation of the hypersurface is $x_{n} f_{k}\left(x_{0}, \ldots, x_{n-1}\right)+$ $f_{k+1}\left(x_{0}, \ldots, x_{n-1}\right)$, where $f_{k}$ is again linear in all variables except at most 4 variables. So the $\mathbb{L}$-rationality follows from Lemma 3.3 and the induction hypothesis.

Remark 4.3. Theorem 4.2 generalizes Corollary 3.3 from AM11, since the equations of the "graph hypersurfaces" considered there are linear in all variables. They must be $\mathbb{L}$-rational by Theorem 4.2 .

Example 4.4 (Affine Potts model hypersurface). The previous theorem also gives an easy way to calculate the modulo $\mathbb{L}$ class of the affine Potts model hypersurfaces appearing in [AM], definition (2.2), equation (2.5). The equations for such affine hypersurfaces are

$$
Z_{G}(q, t)=\sum_{G^{\prime} \subseteq G^{\prime}} q^{k(G)} \prod_{e \in E\left(G^{\prime}\right)} t_{e},
$$

where $G^{\prime}$ is a subgraph of $G$, and $k\left(G^{\prime}\right)$ and $E\left(G^{\prime}\right)$ are the number of connected components and the set of edges of the graph $G^{\prime}$ respectively. Fix $q$ in this equation and denote by $n$ the number of edges of the graph $G$. Then this equation defines the affine Potts model hypersurface in $\mathbb{A}^{n}$. Its class in $K_{0}(V a r)$ is congruent to 1 modulo $\mathbb{L}$ if $n$ is odd and congruent to -1 modulo $\mathbb{L}$ when $n$ is even.

The calculation proceeds as follows. These hypersurfaces are defined by inhomogoneous polynomials of degree $n$ in $\mathbb{A}^{n}$, linear in all variables, where $n$ is the number of the edges of the graph in consideration. Homogenize the equation and write it as $F=0$. Then clearly $F$ is a homogeneous polynomial of degree $n$ linear in all variables except the variable $x_{0}$ introduced in homogenizing. Now the class of the 
affine Potts model hypersurface is $[Z(F)]-\left[Z\left(x_{0}, x_{1} x_{2} \ldots x_{n}\right)\right] . Z(F)$ is $\mathbb{L}$-rational by the previous theorem, and now we are left to calculate $\left[Z\left(x_{0}, x_{1} x_{2} \ldots x_{n}\right)\right]$, which is the class of the union of $n$ hyperplanes in $\mathbb{P}^{n-1}$.

Let $x_{1}, \ldots, x_{n}$ be the projective coordinates of $\mathbb{P}^{n-1}$. Consider the complement of $Z\left(x_{1} x_{2} \ldots x_{n}\right)$, which can be explicitly expressed as points $\left(x_{1}: \cdots: x_{n}\right)$ such that all projective coordinates are nonzero. We see that this affine open set is isomorphic to the $(n-1)$-fold cartesian product of $\left(\mathbb{A}^{1}-\{p t\}\right)$. So the class of the union of $n$ hyperplanes lying in general position in $\mathbb{P}^{n-1}$ equals $\left[\mathbb{P}^{n-1}\right]-(\mathbb{L}-1)^{n-1}$. Taking into account that $\left[\mathbb{P}^{n-1}\right]=1+\mathbb{L}+\cdots+\mathbb{L}^{n-1}$, we see that this class is $1+(-1)^{n}$ modulo $\mathbb{L}$. We conclude that the class of the affine Potts model hypersurface is $\mathbb{L}$-rational when $n$ is odd and congruent to -1 modulo $\mathbb{L}$ when $n$ is even.

\section{REFERENCES}

[AM] Paolo Aluffi and Matilde Marcolli, A motivic approach to phase transitions in Potts models, J. Geom. Phys. 63 (2013), 6-31, DOI 10.1016/j.geomphys.2012.09.003. MR2996396

[AM11] Paolo Aluffi and Matilde Marcolli, Graph hypersurfaces and a dichotomy in the Grothendieck ring, Lett. Math. Phys. 95 (2011), no. 3, 223-232, DOI 10.1007/s11005011-0461-5. MR2775124(2012d:14003)

[Bil] Emel Bilgin, Classes of some hypersurfaces in the grothendieck ring of varieties, arXiv:1112.2131.

[BS] Francis Brown and Oliver Schnetz, A K3 in $\phi^{4}$, Duke Math. J. 161 (2012), no. 10, 1817-1862, DOI 10.1215/00127094-1644201. MR2954618

[BW79] J. W. Bruce and C. T. C. Wall, On the classification of cubic surfaces, J. London Math. Soc. (2) 19 (1979), no. 2, 245-256, DOI 10.1112/jlms/s2-19.2.245. MR533323 (80f:14021)

[CG72] C. Herbert Clemens and Phillip A. Griffiths, The intermediate Jacobian of the cubic threefold, Ann. of Math. (2) 95 (1972), 281-356. MR0302652 (46 \#1796)

[HV92] Kapil H. Paranjape and V. Srinivas, Unirationality of the general complete intersection of small multidegree, Astérisque 211 (1992), 241-248.

[IM71] V. A. Iskovskih and Ju. I. Manin, Three-dimensional quartics and counterexamples to the Lüroth problem, Mat. Sb. (N.S.) 86(128) (1971), 140-166 (Russian). MR0291172 (45 \#266)

[KMM92] János Kollár, Yoichi Miyaoka, and Shigefumi Mori, Rationally connected varieties, J. Algebraic Geom. 1 (1992), no. 3, 429-448. MR.1158625 (93i:14014)

[KR] Bruno Kahn and R. Sujatha, Birational geometry and localisation of categories, arXiv:0805.3753.

[LL03] Michael Larsen and Valery A. Lunts, Motivic measures and stable birational geometry, Mosc. Math. J. 3 (2003), no. 1, 85-95, 259 (English, with English and Russian summaries). MR 1996804 (2005a:14026)

Department of Mathematics, Florida State University, 208 Love Bullding, 1017 ACADemic Way, Tallahassee, Florida 32306

E-mail address: xliao@math.fsu.edu 